Check for updates

Cite this: RSC Adv., 2019, 9, 11212

\title{
Tunable porous silica nanoparticles as a universal dye adsorbent
}

\begin{abstract}
S. Irudhaya Raj, (D) a Adhish Jaiswal (DD *a and Imran Uddin (DD ${ }^{\text {b }}$
Here, we report selective adsorption of cationic dyes methylene blue (MB) and rhodamine $B(R B)$ and anionic dyes methyl orange (MO) and bromo cresol green (BCG) by modifying the surface of cetyl trimethyl ammonium bromide (CTAB) coated porous silica nanoparticles (PSN). We used a top down approach to synthesize PSN (porous silica nanoparticles) without high temperature calcination. X-ray diffraction study confirms the formation of pure phase silica nanoparticles. SEM analysis reveals that the particle morphology is spherical and the size range lies in-between 150-200 nm. We have studied the dye adsorption properties for three cases of PSN at varying calcination temperatures of $100{ }^{\circ} \mathrm{C}, 250{ }^{\circ} \mathrm{C}$ and $500{ }^{\circ} \mathrm{C}$, respectively. Thermal study has been performed in the temperature range of $50-800{ }^{\circ} \mathrm{C}$ to check the calcination temperature. In this report, we have tuned the surface properties for selective adsorption of cationic and anionic dyes in water. In the first case, $100{ }^{\circ} \mathrm{C}$ calcined PSN selectively adsorb only anionic dyes, whereas in the second case, $500{ }^{\circ} \mathrm{C}$ calcined PSN adsorb only cationic dyes and finally, an optimized calcination temperature $\approx 250{ }^{\circ} \mathrm{C}$ could be used for all types of dye to be adsorbed irrespective of charges on the dyes. The mode of interaction of dyes with PSN has been explained with a proper mechanism in all three cases. The adsorptions of dyes are confirmed by UV-Vis spectroscopy. Adsorption capacity and regenerable performance of adsorbents have also been studied.
\end{abstract}

Received 20th December 2018

Accepted 3rd April 2019

DOI: $10.1039 / \mathrm{c} 8 \mathrm{ra10428j}$

rsc.li/rsc-advances usage of adsorbents is an ideal option for remediation of coloured waste water. ${ }^{6}$ Activated carbon has been used as commercial adsorbent for remediation of waste water for many decades due to its high removal efficiency and capacity. However, its cost, regeneration difficulties, use of carbon source and carbon emission associated with the production and application of activated carbon has been a challenge. Therefore, there is a need for low cost, environment friendly materials, regenerable and reusable adsorbents for remediation of industrial waste water.

The silica nanoparticles have attracted significant attention as nano adsorbent on account of their regular structural characteristics, larger surface area, tunable pore diameters, good thermal and chemical stabilities, ease of surface modification, selectivity of organic pollutants, economical regeneration and reusability. ${ }^{7,8}$ Morphology controlled nanomaterials like hollow, mesoporous and tunable nanoporous silica nanoparticles play crucial role in drug delivery application. ${ }^{9-11}$ Therefore finetuning of particle size, surface functionalization and porous structure is very critical for environmental and medical applications. ${ }^{\mathbf{1 2 - 1 5}}$ Obviously, the final porous structure, morphology, dimension and surface property of the silica nanoparticles are highly dependent on various reaction parameters such as solvent, temperature, $\mathrm{pH}$, surfactant/silica molar ratio, cosurfactant and stirring rates..$^{\mathbf{8}, 16,17}$

Templating of silica nanoparticles with cationic surfactants, is very typical due to the electrostatic interaction between 
cationic head group of surfactants and negative surface of silica, further the silica self assembles around the template producing ordered channels. ${ }^{18,19}$ The cationic surfactant CTAB acts as dispersing and pore templating agent; however, its concentration regulates the final porous framework. ${ }^{7,19}$ The surfactant template on silica nanoparticles acts as a hydrophobic site around the silica surface, forming a mono, bi or aggregates depending on the concentration of surfactant. ${ }^{20-22}$ The real structure of CTAB templated silica surface is more complex and intriguing. ${ }^{23,24}$ The interfacial properties of the silica and surfactant layer plays a vital role in the adsorption of dyes. Bryleva et al. demonstrated the behaviour of different indicator dyes in CTAB modified silica nanoparticles and confirmed the existence of similarity between CTAB templated silica nanoparticles and common spherical surfactant micelle. ${ }^{21}$ Biggs et al. have reported that above, the critical micelle concentration (CMC) the micelle directly adsorbs on the silica surface. ${ }^{25}$ The adsorbed CTAB layer has considerable attraction and repulsion to counter ions present in the medium. Generally, the 'assynthesized' silica nanoparticles retain the surfactant template within the silica network. However, if the surfactant molecules are not needed, it can be removed by calcination in air at or above $500{ }^{\circ} \mathrm{C}$, solvent extraction or microwave digestion. ${ }^{7}$ In this context, silica nanoparticles templated with surfactant layer are removed to open the pores for dye adsorption, ${ }^{\mathbf{8}, 14}$ enzyme immobilization, ${ }^{14}$ surface functionalisation ${ }^{13}$ and drug delivery.$^{13}$ It is also noteworthy that uncalcined mesoporous silica materials are used as efficient adsorbent for removal of organic pollutants from the aqueous media. ${ }^{7,26,27}$ Hence, it is of significant interest to examine the effect of surfactant template and calcination temperatures in the dye adsorption behavior of silica nanoparticles. However, the report about the structural evolution during the surfactant removal process is scarce; hence it is of significant interest to examine the role and mechanism of dye adsorption by silica nanoparticles in detail.

In our present work, porous silica nanoparticles (PSN) with tunable surface properties were synthesized by top-down approach using silica gel as precursor and CTAB as template. Further, the as-synthesized PSN were calcined at various temperatures to remove the partial and complete surfactant layer from the PSN surface. A detailed study on dye adsorption behavior by the as-synthesized PSN particles calcined at $100{ }^{\circ} \mathrm{C}$, $500{ }^{\circ} \mathrm{C}$ and optimized $\approx 250{ }^{\circ} \mathrm{C}$ and their mechanistic path of interaction has been reported. The calcined products were tuned according to their specific properties and structure for the desired application. Our insight into the structural evolution and removal of surfactant layer were investigated using scanning electron microscopy (SEM) and thermo-gravimetric analysis (TGA). Furthermore, the selective dye adsorption, adsorption efficiency and regeneration and reusability of adsorbent were confirmed by a series of adsorption experiments.

\section{Materials \& methods}

All of the chemicals were of an analytical grade and purchased from Merck Ltd. with more than $98 \%$ purity. The aqueous solutions were prepared with double distilled water for all of the experiments.

\section{Top down approach}

In the typical synthesis, the stock silica gel solution was prepared by mixing of silica gel self-indicating coarse $(1.0 \mathrm{~g})$ with $70 \mathrm{~mL}$ of ammonia solution and heated to $80^{\circ} \mathrm{C}$, the silica gel turned blue with popping sound and became soluble. In another beaker CTAB ( $2 \mathrm{~g}, 0.005$ moles) was mixed in water (50 $\mathrm{mL})$ at room temperature with constant stirring. The prepared silica gel stock solution $(30 \mathrm{~mL})$ was slowly mixed with СТАВ solution $(50 \mathrm{~mL})$, and then the reaction mixture was stirred, heated to $60{ }^{\circ} \mathrm{C}$ for $30 \mathrm{~min}$ and aged for $24 \mathrm{~h}$. After aging, the flocculated PSNs were isolated by centrifugation and dried at room temperature for $12 \mathrm{~h}$. Finally, the samples were calcined at $100{ }^{\circ} \mathrm{C}, 250{ }^{\circ} \mathrm{C}$ and $500{ }^{\circ} \mathrm{C}$ for the partial, optimized and complete removal of CTAB template for the desired application. Hereafter, we refer to the individual samples as PSN-100, PSN250 and PSN-500.

\section{Results and discussion}

We speculate that the possible formation mechanism of PSNs involves electrostatic ion interaction. In our typical synthesis procedure, micron size porous silica gel reacted with ammonia solution at a certain temperature leading to the formation of silica-ammonia complex. ${ }^{28}$ Further, on heating silica-ammonia complex with CTAB solution, the ammonia molecules were expelled out, thereby polar head group of CTAB were attached over the negatively charged silica surface. Therefore, electrostatic force of attraction is the operating mechanism for the formation of PSN.

\section{Phase \& morphology}

The as-synthesized PSN ware characterized by XRD techniques for the exact phases and purity of the samples as shown in Fig. 1. Powder X-ray diffraction pattern (XRD) of the assynthesized PSN were recorded by a PANalytical X'PERT PRO instrument using an iron-filtered $\mathrm{Cu}-\mathrm{K} \alpha$ radiation $(\lambda=1.5406 \AA)$

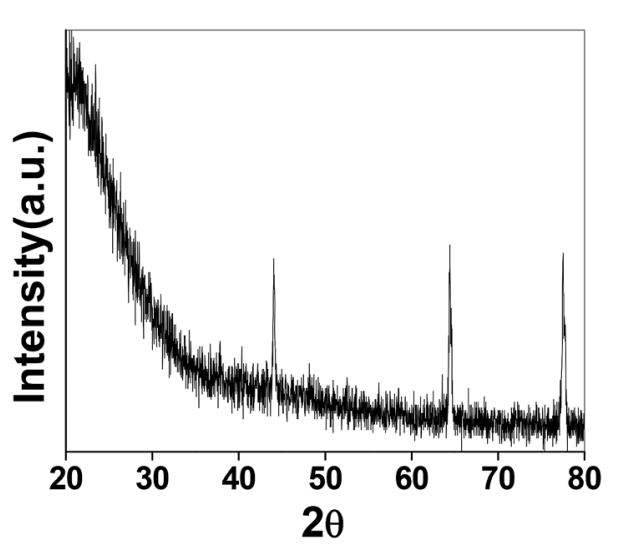

Fig. 1 Room temperature powder XRD pattern of porous silica nanoparticles (PSN). 
in the $2 \theta$ range of $10-80^{\circ}$ with a step size of $0.02^{\circ}$. Phase and purity was confirmed by the reference taken from JCPDS card no. 421401.

Further size and morphology were characterised through SEM analysis. The SEM analysis on the samples was done using the Quanta 200 3D from FEI operating at $20 \mathrm{kV}$ energy. Fig. 2A-C shows SEM images of PSN-100, PSN-250 and PSN-500 particles, respectively. A detailed SEM investigation of particle surfaces indicates that PSN-100 particles were spherical in shape, size inbetween the range of 150-200 $\mathrm{nm}$ and well separated. After calcination treatment around $250{ }^{\circ} \mathrm{C}$ and $500{ }^{\circ} \mathrm{C}$, spherical particles were started to fuse together to form agglomerated larger size particles. It is clear that as the calcinations temperature increases, agglomeration increases due to the removal of CTAB layer from the surface of PSN.

Furthermore, TGA analysis was performed by PerkinElmer Pyris TGA instrument for PSN-100 particles between the temperature range $50-800{ }^{\circ} \mathrm{C}$. In Fig. 3 , TGA study shows a small initial drop in weight from $50-100{ }^{\circ} \mathrm{C}$. This initial drop in weight is generally credited to the release of water adsorbed on the surface. After the initial drop, a sharp decrease in weight occurs until approximately $250{ }^{\circ} \mathrm{C}$. This sharp decrease in weight can be associated with the partial removal of CTAB structure from the surface. With increasing temperature, a gradual decrease in weight exist up to a limit of $800{ }^{\circ} \mathrm{C}$, this can be related with the complete removal of CTAB entity from the surface.

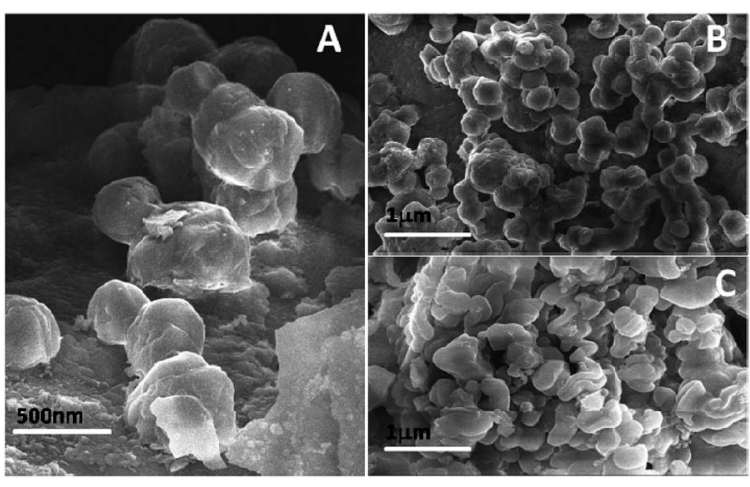

Fig. 2 SEM images of (A) PSN-100, (B) PSN-250 and (C) PSN-500.

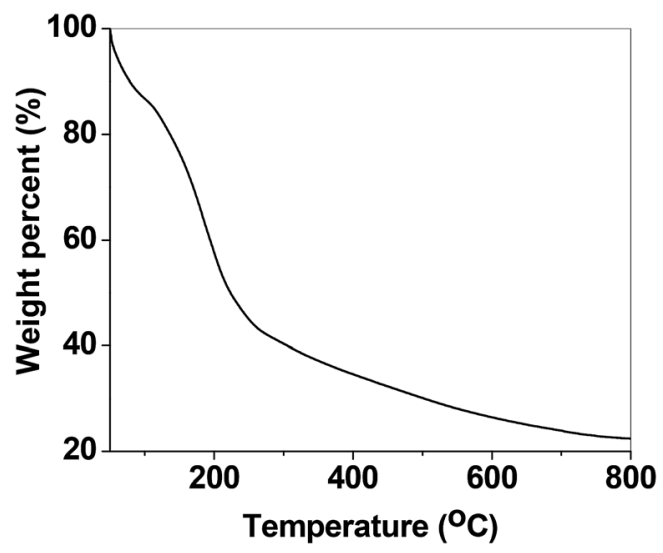

Fig. 3 TGA graph of PSN-100.
Generally, porous materials have shown good adsorption property for the removal of dyes. Therefore, we had performed experiments of dye adsorption by using the synthesized porous materials (PSN-100, PSN-500 and PSN-250) which were monitored using UV-Vis spectrophotometer (Shimadzu, UV-1800) over a scanning range from $190-800 \mathrm{~nm}$ at resolution of $2 \mathrm{~nm}$.

\section{Dyes adsorption}

All the adsorption experiments were carried out at room temperature. First, we have selected four sample dyes to test the selective adsorption performance of absorbent: methyl orange (MO), bromocresol green (BCG), Methylene Blue (MB) and Rhodamine B (RB), respectively. MO and BCG are water soluble anionic dyes, whereas $\mathrm{RB}$ and $\mathrm{MB}$ are water soluble cationic dyes. In a series of typical experiments, $10 \mathrm{mg}$ each of PSN-100, PSN-250 \& PSN-500 was soaked into $10 \mathrm{~mL}$ each of four dyes with concentration $300 \mathrm{mg} \mathrm{L}^{-1}$ and $\mathrm{pH}$ value was between 6.5 and 7.5. After certain time intervals, we have collected absorption data by using UV-Vis Spectrophotometer.

The experimental results of selective adsorption experiment using four sample dye solutions using PSN-100, 500 \& 250 adsorbents has been shown in Fig. 4. Firstly, we observed that the color of MO and BCG solutions before and after PSN100 adsorption changed from orange, pale blue to transparency, whereas no substantial color change was observed for $\mathrm{RB}$ and $\mathrm{MB}$ as shown in Fig. 4B, indicating the adsorption of only anionic dyes. Secondly, in PSN-500 it was observed that color of $\mathrm{RB}$ and $\mathrm{MB}$ solutions before and after adsorption changed from purple, blue to transparency, whereas no substantial color change for $\mathrm{MO}$ and $\mathrm{BCG}$, indicating the adsorption of cationic dyes as shown in Fig. 4C. Finally, in the optimized case of PSN-250 adsorbent, the color of MO, BCG, $\mathrm{RB}$ and $\mathrm{MB}$ solutions before and after adsorption changed from orange, pale blue, purple and blue to transparency, indicating the adsorption of both anionic and cationic dyes as shown in Fig. 4D.

\section{Confirmation of dye adsorption by UV-Vis spectroscopy.}

The adsorption of dyes by the different synthesized adsorbents was further checked by UV-Vis spectroscopy. From Fig. 5, it can be seen that the drop in concentration of dyes with time was observed for MO and BCG with PSN-100; RB and MB with PSN500; MO, BCG, RB and MB with PSN-250 adsorbents. In all the adsorption experiments, the drop in absorbance maxima for MO, BCG, RB and MB were analyzed at 464, 617, 561 and $661 \mathrm{~nm}$, respectively. Spectroscopic study also confirmed that only anionic dyes were absorbed by PSN-100 and cationic dyes were absorbed by PSN-500, whereas both the dyes were absorbed by PSN-250. Those dyes which were not adsorbed by adsorbents the change in absorption spectra remains insignificant. Both spectroscopic as well as visual detection experiments clearly validate the selective adsorption of anionic, anionic and cationic, and cationic dyes by PSN-100, optimized PSN-250 and PSN-500 adsorbents, respectively. 


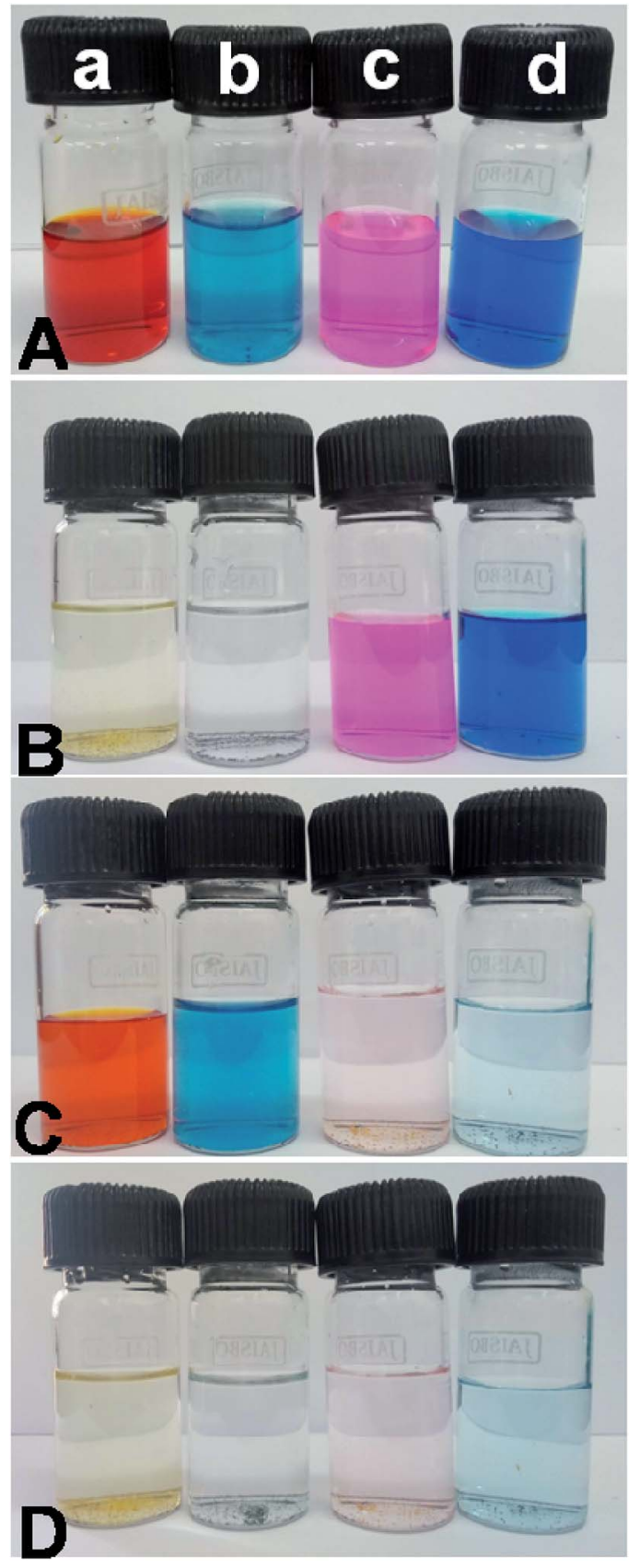

Fig. 4 (A) Shows color of dyes (a) MO, (b) BCG, (c) RB and (d) MB before adsorption, whereas ( $B, C$ and $D)$ shows colour of dyes after adsorption by PSN-100, PSN-500 and PSN-250, respectively.

\section{Adsorption mechanism}

It is essential to identify the structural evolution and possible mechanism for dye adsorption by synthesized adsorbents. The adsorption performance of an adsorbent is highly dependent on its surface area, pore size, pore volume and its selectivity depends on its surface charge and electrostatic attraction. The mechanism of adsorption of surfactant on silica surface can be summarised in the following steps: in the first step, when small quantity of CTAB molecule interact with silica particle, there is a formation of surfactant monolayer by electrostatic attraction between the positively charged head group of surfactants and
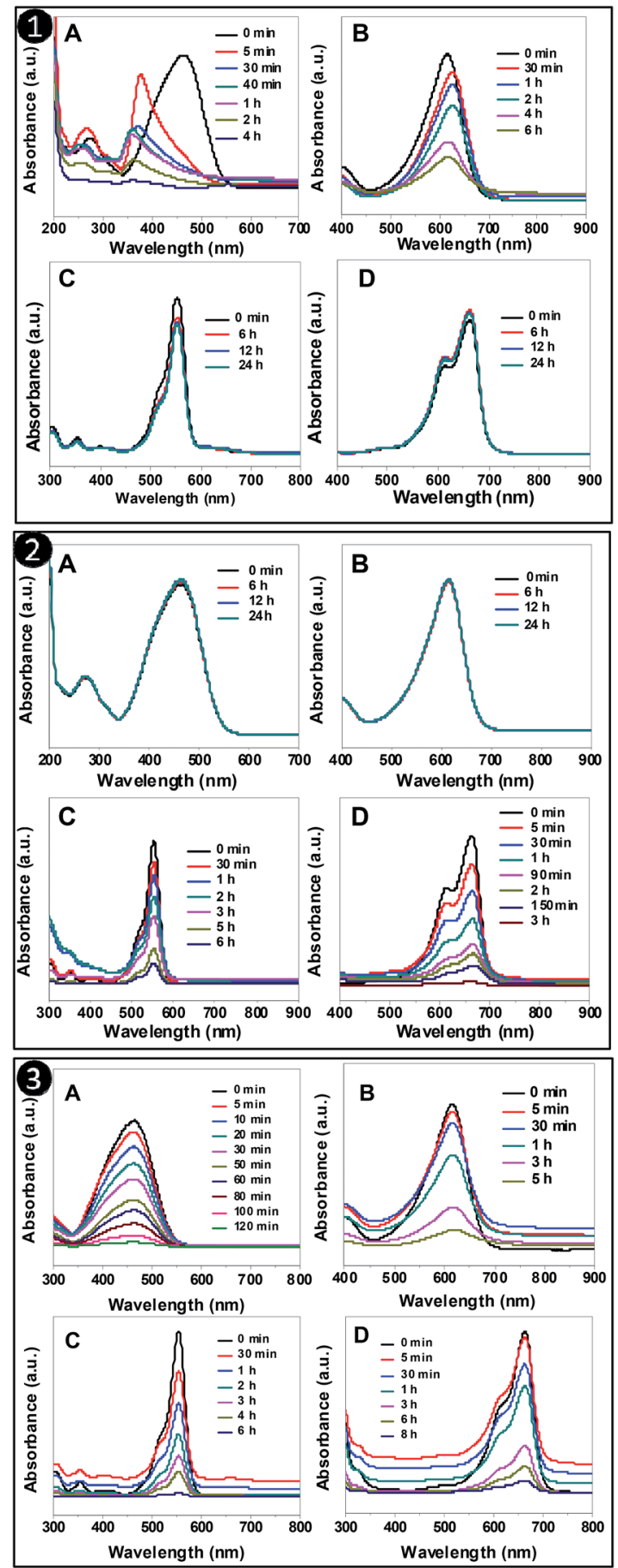

Fig. 5 UV-Vis absorption spectra of organic dyes (A) MO (B) BCG (C) $\mathrm{RB}$ (D) MB after interaction with: (1) PSN-100 (2) PSN-500 and (3) PSN250 at different time interval.

negative charged silica surface. This result in the formation of PSN stabilized by hydrophobic environment. In the second step, after the addition of slight excess of CTAB (more than CMC) there would be formation of surfactant bilayer through the interaction of hydrophobic tail group of CTAB molecules which results, the formation of positive charge on surface of PSN. ${ }^{20-22}$

It is already reported that during the process of calcination the surfactant layer would be exfoliated slowly which favor 
particle aggregation and pore size growth. On the basis of this hypothesis and experimental results, we speculate the possible dye adsorption mechanism and structural evolution for PSN100, PSN-250 and PSN-500 adsorbent into three stages as shown in Fig. 6. In the first stage, the calcination of PSN at $100{ }^{\circ} \mathrm{C}$ would have positive surface charge with surfactant bilayers as shown in Fig. 6A. Therefore in PSN-100, electrostatic attraction between positively charged adsorbent and anionic dyes was primarily responsible for adsorption of anionic dyes. In the second stage, the calcination process at or above $500{ }^{\circ} \mathrm{C}$ removed the surfactant layer completely from the silica surface and left negatively charged silica particles. Moreover, the
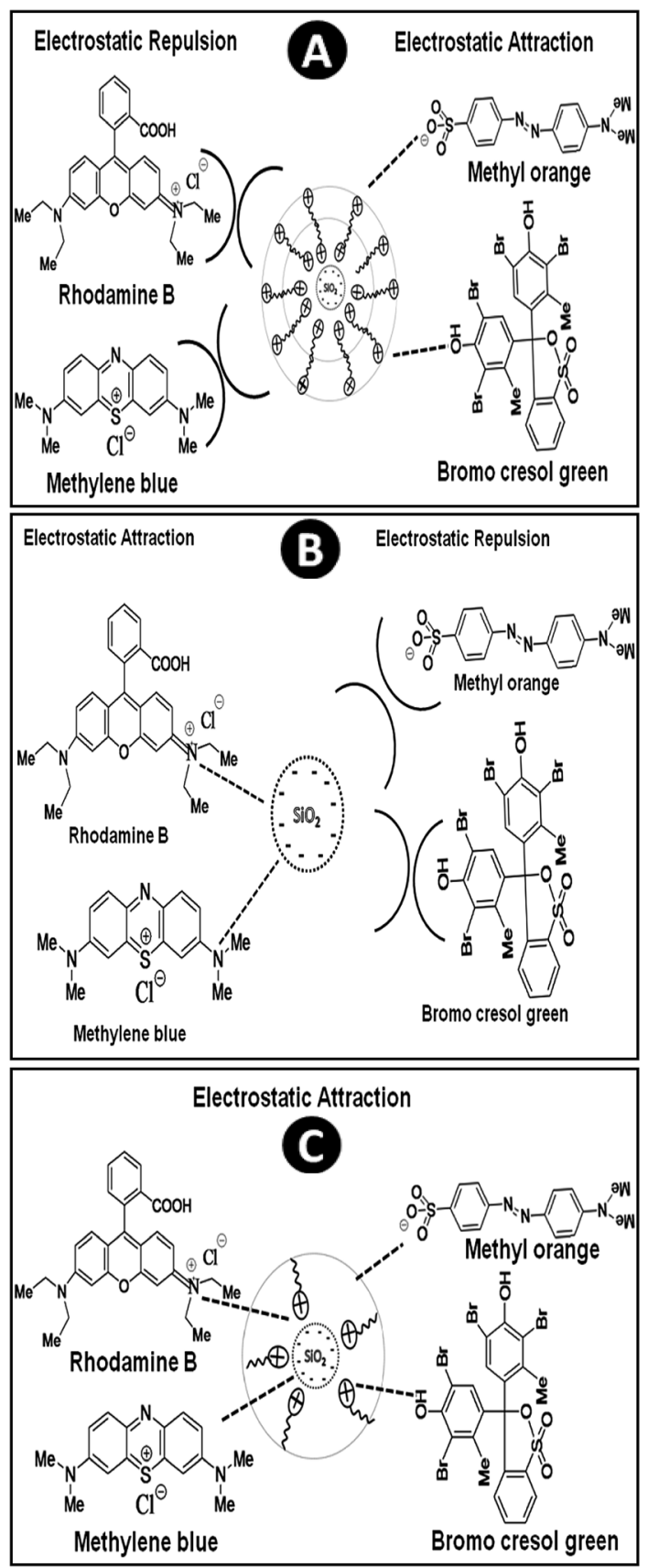

Fig. 6 Proposed mechanism of interaction of organic dyes with: (A) PSN-100 (B) PSN-500 and (C) PSN-250. electrostatic attraction between the negative and positive surface charge of adsorbent and dyes were key driving force for the adsorption of cationic dyes as shown in Fig. 6B. In third stage, the optimized calcination $\left(240-250{ }^{\circ} \mathrm{C}\right)$ have resulted to partial removal of surfactant bilayer which favour adsorption of both cationic and anionic dyes, this peculiar property could be explained by the electrostatic attraction as shown in Fig. 6C. TGA study also confirms that there is loss in weight and partial removal of surfactant layer above $200{ }^{\circ} \mathrm{C}$ which will open the door for adsorption of both anionic \& cationic dyes. Moreover, the surfactant bilayer would not be much stable above $200{ }^{\circ} \mathrm{C}$ and it would favor the aggregation of the PSN particles and minimize the repulsion of cationic head group of surfactant and cationic dyes to a certain extent. This leads to adsorption of both cationic dyes and anionic dyes with PSN-250 adsorbent.

\section{MO-CTAB interaction}

We have observed interesting facts during the adsorption of dyes that absorption spectra of dyes showed blue shift after interaction with cationic part of the surfactant. To deeply evaluate the interaction, fixed concentration $\left(1.0 \times 10^{-5} \mathrm{M}\right)$ of MO was added into varying concentration of CTAB solution and absorbance spectra was recorded using UV-Vis spectroscopy. We observed a pronounced blue shift in $\lambda_{\max }$ of MO from $464 \mathrm{~nm}$ to $375 \mathrm{~nm}$. Similar behavior was also observed by various research groups. ${ }^{29-31}$ In Fig. 7 we have shown that as the concentration of CTAB increases in the mixture of MO-CTAB solution, $\lambda_{\max }$ shifted from $460 \mathrm{~nm}$ to $375 \mathrm{~nm}$ between the concentration range of $10^{-4}$ to $10^{-5} \mathrm{M}$; furthermore, it reached $420 \mathrm{~nm}$ at much higher concentration range of $10^{-3}$ to $10^{-1} \mathrm{M}$.

Hence, the blue shift spectral signature as depicted in Fig. 7 was an affirmation for the interaction of MO with the CTAB templated PSN adsorbents. We further examined the tunability of emission colour of MO-CTAB mixture under UV light at $365 \mathrm{~nm}$ as shown in Fig. 8B. We observed greenish colour at wide range of varying concentration of CTAB from $1 \times 10^{-8} \mathrm{M}$ to $1 \times 10^{-1} \mathrm{M}$, but exceptionally transparent at $1 \times 10^{-4} \mathrm{M}$.

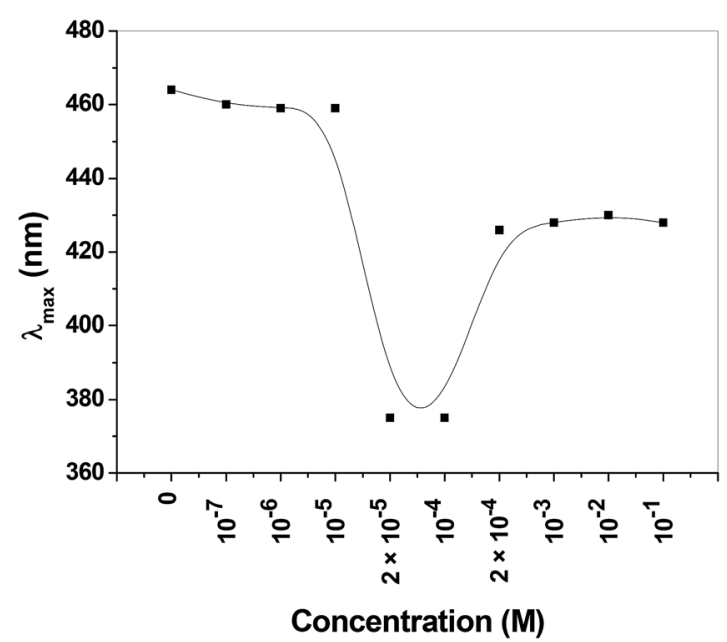

Fig. 7 Behavior of absorption maxima $\left(\lambda_{\max }\right)$ of methyl orange after mixing with various concentration of CTAB. 
This might be due to the cis-trans-cis photo isomerization of azo bond of $\mathrm{MO}$ and change in micro-environment of dye in micellar medium. ${ }^{31}$ The change in emission colour of MOCTAB mixture required further study using various other tools, which was beyond the scope of our present experiment.

\section{Adsorption capacity and dye removal efficiency}

Based on the results of adsorption studies, we carried out batch experiments to test the adsorption capacity and dye removal efficiency of PSN-100, PSN-250 \& PSN-500. In set of parallel experiments, $50 \mathrm{mg}$ of respective adsorbents were added into $50 \mathrm{~mL}$ respective aqueous dye solutions as follows: PSN-100 with MO and BCG; PSN-250 with MO, BCG, RB and MB; PSN500 with $\mathrm{RB}$ and $\mathrm{MB}$ with variable initial concentration (100, 200, 300, 400, $500 \mathrm{mg} \mathrm{L}^{-1}$ ). Next, the adsorbent-dye mixtures was stirred for a certain time and left undisturbed for $24 \mathrm{~h}$. After reaching equilibrium, the adsorbents was separated from the dye solutions by filtration and the remaining concentration of dyes in the filtrates was determined by using a UV-Vis spectrophotometer.

The adsorption capacity of adsorbent $\left(Q_{\mathrm{e}} \mathrm{mg} \mathrm{g}^{-1}\right)$ was calculated using the following equation:

$$
Q_{\mathrm{e}}=\frac{\left(C_{\mathrm{o}}-C_{\mathrm{e}}\right) V}{W}
$$

The dye removal efficiency at equilibrium time was calculated using the following equation:

$$
\% \text { removal efficiency }=\frac{C_{\mathrm{o}}-C_{\mathrm{e}}}{C_{\mathrm{o}}} \times 100
$$

where $C_{\mathrm{o}}$ and $C_{\mathrm{e}}$ are initial and equilibrium concentrations of dyes ( $\left.\mathrm{mg} \mathrm{L}^{-1}\right), V$ is the volume of the solution (L), and $W$ is the mass of adsorbent used $(\mathrm{g})$ in the experiment.

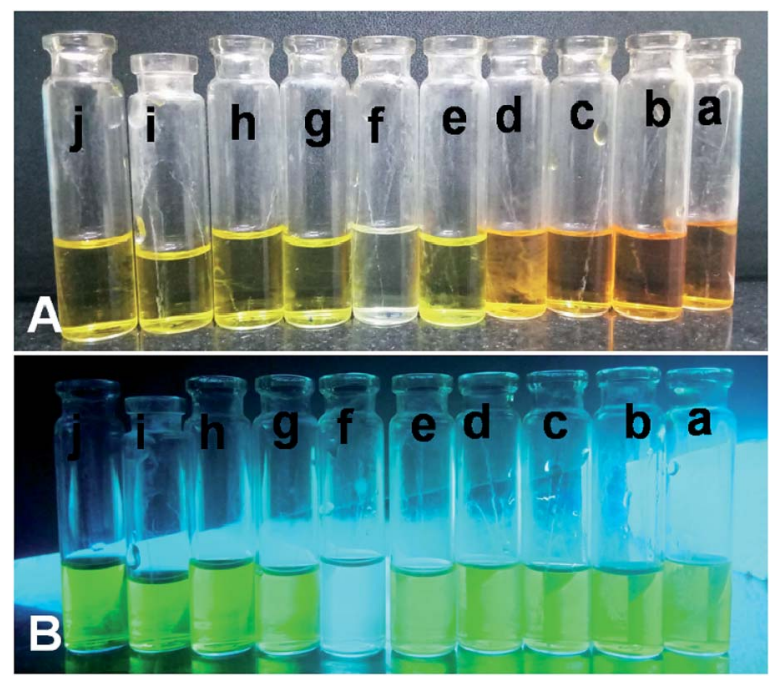

Fig. 8 Photographs of (A) day light and (B) UV light (365 nm) illumination on $\mathrm{MO}\left(1.0 \times 10^{-5} \mathrm{M}\right)$ with varying $\mathrm{CTAB}$ concentration: (a) $10^{-8} \mathrm{M}$ (b) $10^{-7} \mathrm{M}$ (c) $10^{-6} \mathrm{M}$ (d) $10^{-5} \mathrm{M}$ (e) $5 \times 10^{-5} \mathrm{M}$ (f) $1 \times 10^{-4} \mathrm{M}$ (g) $5 \times 10^{-4} \mathrm{M}$ (h) $10^{-3} \mathrm{M}$ (i) $10^{-2} \mathrm{M}$ and (j) $10^{-1} \mathrm{M}$.
Calibration experiments were performed to assess the amount of adsorbed dye after a given time using the absorbance of the standard aqueous solutions of dyes. Based on eqn (1) after $24 \mathrm{~h}$, the equilibrium concentration $\left(Q_{\mathrm{e}}\right)$ were as follows: MO and BCG on adsorbent PSN-100 were $295.05 \mathrm{mg} \mathrm{L}^{-1}$ and $297.54 \mathrm{mg} \mathrm{L}^{-1}$; MO, BCG, RB and MB on adsorbent PSN-250 were $271 \mathrm{mg} \mathrm{L}^{-1}, 272 \mathrm{mg} \mathrm{L}^{-1}, 274 \mathrm{mg} \mathrm{L}^{-1}$ and $277 \mathrm{mg} \mathrm{L}^{-1}$; $\mathrm{RB}$ and $\mathrm{MB}$ on adsorbent $\mathrm{PSN}-500$ were $286 \mathrm{mg} \mathrm{L}^{-1}$ and $290 \mathrm{mg} \mathrm{L}^{-1}$ for respective dyes as shown in Fig. 9A. Based on eqn (2) the adsorption percentage were as follows: MO and BCG on adsorbent PSN-100 were 98.35\% and 99.18\%; MO, BCG, RB and $\mathrm{MB}$ on adsorbent PSN-250 were 90.46\%, 90.58\%, 91.56\%
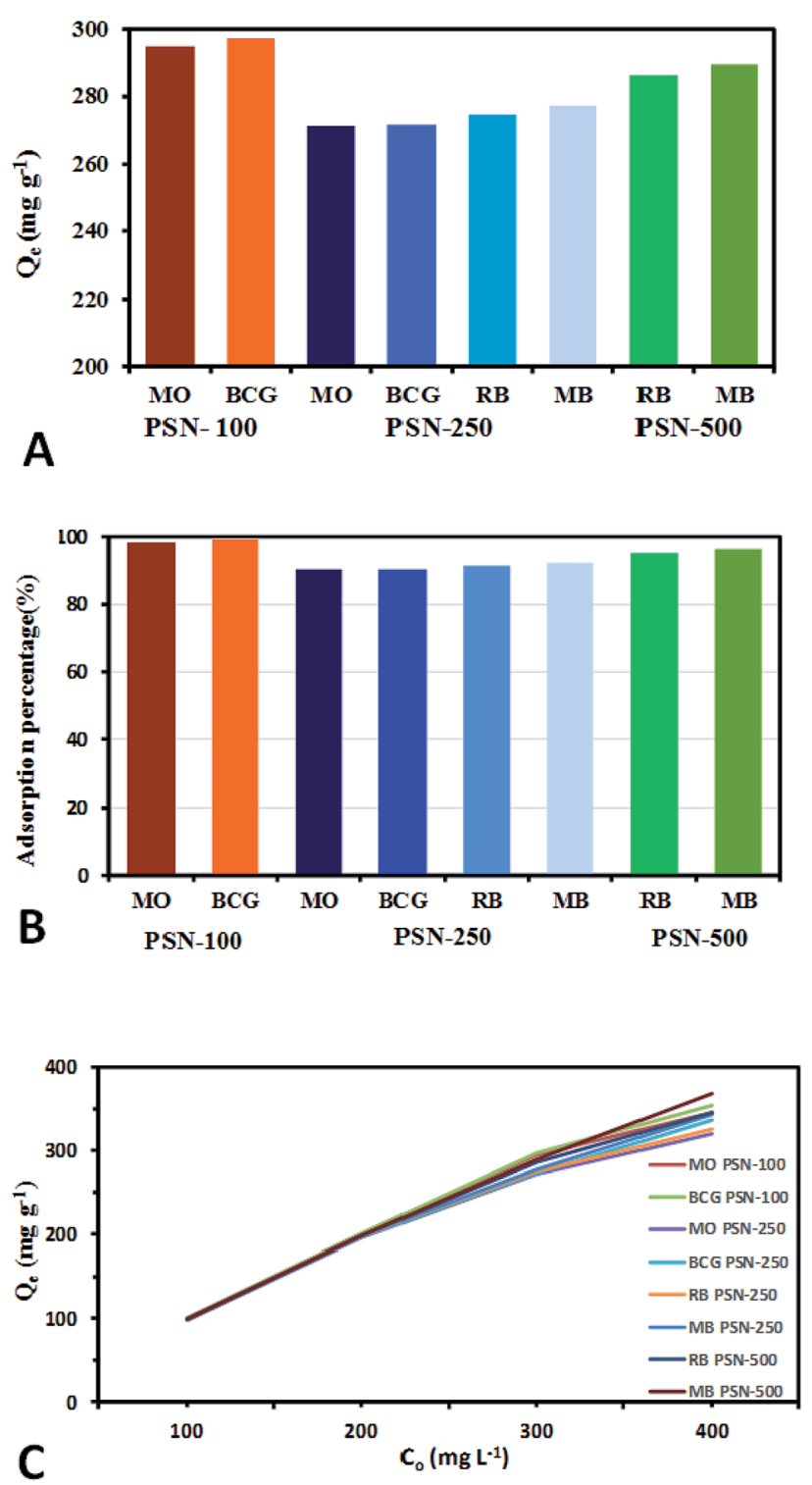

Fig. 9 A) Equilibrium concentration of dyes on PSN-100, 250 and 500 adsorbent after $24 \mathrm{~h}$ (initial concentration: $10 \mathrm{mg}$ adsorbents, $300 \mathrm{mg} \mathrm{L}^{-1}$ dyes). (B) Adsorption percentage of dyes on PSN-100, 250 and 500 adsorbent after $24 \mathrm{~h}$ (initial concentration: $10 \mathrm{mg}$ adsorbents, $300 \mathrm{mg} \mathrm{L}^{-1}$ dyes). (C) Effect of initial concentration of dyes on adsorbents samples. (initial concentration: $50 \mathrm{mg}$ adsorbents, 100$400 \mathrm{mg} \mathrm{L}^{-1}$ dyes). 
and $92.43 \%$; $\mathrm{RB}$ and $\mathrm{MB}$ on adsorbent PSN-500 were $95.45 \%$ and $96.5 \%$, respectively as shown in Fig. 9B. With the initial increase in concentration from 100 to $400 \mathrm{mg} \mathrm{L}^{-1}$, the equilibrium adsorptions $\left(Q_{\mathrm{e}}\right)$ gradually increases, and the maximum adsorptions for MO, BCG on PSN-100; MO, BCG, RB, MB on PSN-250; RB, MB on PSN-500 were 345.67, 354.52, 320.49, $336.67,325.42,342.65,345.08$ and $368.68 \mathrm{mg} \mathrm{g}^{-1}$, respectively as shown in Fig. 9C. All the above mentioned experimental findings indicate calcination triggered versatile, tunable and selective dye adsorption property of synthesized adsorbent.

\section{Regeneration and reusability of adsorbent}

For readsorption experiments, $50 \mathrm{mg}$ of PSN-100, 250 and 500 samples loaded with dyes was washed by desorption solutions, dried and redisposed into $50 \mathrm{~mL}$ of dye solution with concentration $300 \mathrm{mg} \mathrm{L}^{-1}$. An important property of adsorbent is to possess good sorption and excellent regenerative capacity. In brief, after dye adsorption, anionic dye (MO \& BCG) adsorbed by PSN-100 and PSN-250, cationic dye (RB \& MB) adsorbed by PSN250 and PSN-500 were collected and washed with distilled water for removing the unabsorbed dyes. After washing, the dye loaded PSN-100, PSN-250 \& PSN-500 samples were soaked in $10 \mathrm{~mL}$ of desorption solutions for $1 \mathrm{~h}$ and agitated at $400 \mathrm{rpm}$ for $10 \mathrm{~min}$. For anionic dyes (MO and BCG) basic medium of aqueous $\mathrm{NH}_{3}$ in $50 \%(\mathrm{v} / \mathrm{v})$ ethanol, while for cationic dyes (RB and $\mathrm{MB}$ ) acidic medium of $0.5 \mathrm{M} \mathrm{HCl}$ in $50 \%(\mathrm{v} / \mathrm{v})$ ethanol was selected as the desorption solution. In basic $\mathrm{pH}$, increase in number of hydroxyl ions, destabilizes the electrostatic attraction between positively charged surface of surfactant and the anionic dyes. While in acidic $\mathrm{pH}$, the hydrogen ions stabilizes the negatively charged surface of silica and provides favorable environment for desorption of cationic dyes. This result indicated that main chemical reaction during the adsorption/ desorption is the electrostatic ion mechanism. Finally the regenerated PSN-100, 250 \& 500 were dried and reused in the next cycle of dye adsorption experiments.

The adsorption-desorption experiments have been shown in Fig. 10. We observed the adsorption capacity of PSN-100, PSN-250 \& PSN-500 decreased after five, three and five consecutive cycles respectively and the removal efficiency was still greater than $90 \%$ for PSN-500 \& 100, while for PSN-250 a slight decrease around 85$90 \%$ after third cycle. We speculate that the decrease in efficiency of PSN-500 and PSN-250 was due to pore filling mechanism,

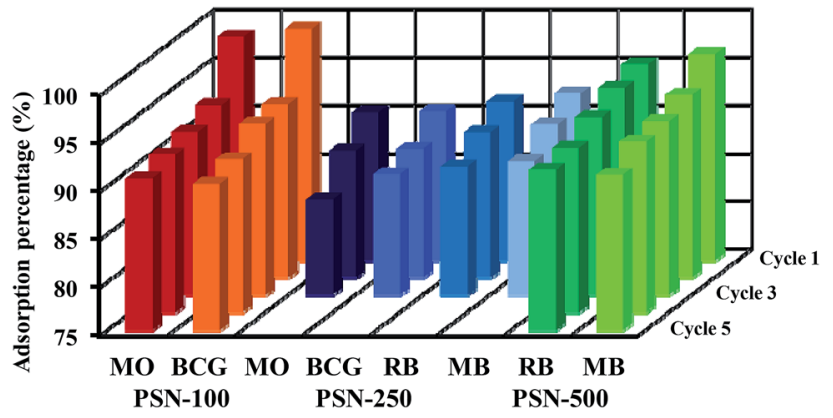

Fig. 10 The regenerable performance of adsorbents. whereas for PSN-100 was due to destabilization of surfactant layer. Therefore, the prepared adsorbents has a good versatility in dye adsorption, regeneration and reusability which is suitable for coloured waste water cleanup with high-efficiency.

\section{Conclusion}

In summary, CTAB coated PSN particles were successfully synthesized by top down approach by controlled calcination. The, CTAB template has considerable influence on adsorption of dyes has been demonstrated. Experimental results indicated that tunable morphology of porous silica nanoparticles showed selective adsorption of cationic and anionic dyes with specificity: (1) PSN-100 showed specificity towards anionic dyes (2) PSN-500 showed specificity towards cationic dyes (3) PSN-250 showed specificity towards both cationic and anionic dyes. Mode of interaction of dyes and its selectivity with PSN have been successfully explained through the electrostatic interaction. Adsorption-desorption experiment has been performed to check its regenerable ability and reusability. Further, PSN adsorption behaviour can be exploited in medical and biomedical applications. We have to work on the fluorescence property of MO in micellar medium, mechanistic path of formation of PSN and adsorption isotherm in more details, which will further help to explore this field.

\section{Conflicts of interest}

There are no conflicts to declare.

\section{Abbreviations}

$\begin{array}{ll}\text { CTAB } & \text { Cetyl trimethyl ammonium bromide } \\ \text { MO } & \text { Methyl orange } \\ \text { BCG } & \text { Bromo cresol green } \\ \text { MB } & \text { Methylene blue } \\ \text { RB } & \text { Rhodamine B } \\ \text { CMC } & \text { Critical micelle concentration } \\ \text { PSN } & \text { Porous silica nanoparticles } \\ \text { PSN-100 } & \text { Porous silica nanoparticles calcined } 100{ }^{\circ} \mathrm{C} \\ \text { PSN-250 } & \text { Porous silica nanoparticles calcined } 250{ }^{\circ} \mathrm{C} \\ \text { PSN-500 } & \text { Porous silica nanoparticles calcined } 500{ }^{\circ} \mathrm{C}\end{array}$

\section{Acknowledgements}

Adhish Jaiswal and Raj acknowledge financial support from IGN Tribal University, Amarkantak and Raj acknowledges the IGNTU for providing research fellowship.

\section{References}

1 A. Ahmad, S. H. Mohd-Setapar, C. S. Chuong, A. Khatoon, W. A. Wani, R. Kumar and M. Rafatullah, RSC Adv., 2015, 5, 30801-30818.

2 A. Bafana, S. S. Devi and T. Chakrabarti, Environ. Rev., 2011, 19, 350-371. 
3 S. P. S. Markandeya and D. Mohan, Res. J. Environ. Toxicol., 2017, 11, 72-89.

4 L. Labiadh, A. Barbucci, M. P. Carpanese, A. Gadri, S. Ammar and M. Panizza, J. Electroanal. Chem., 2016, 766, 94-99.

5 K. Lokesh and R. Sivakiran, J. Biochem. Technol., 2014, 3, 177-180.

6 M. T. Yagub, T. K. Sen, S. Afroze and H. M. Ang, Adv. Colloid Interface Sci., 2014, 209, 172-184.

7 L. T. Gibson, Chem. Soc. Rev., 2014, 43, 5173-5182.

8 S. Kachbouri, N. Mnasri, E. Elaloui and Y. Moussaoui, J. Saudi Chem. Soc., 2018, 22, 405-415.

9 Y. Li, N. Li, W. Pan, Z. Yu, L. Yang and B. Tang, ACS Appl. Mater. Interfaces, 2017, 9, 2123-2129.

10 R. K. Singh, K. D. Patel, K. W. Leong and H. W. Kim, ACS Appl. Mater. Interfaces, 2017, 9, 10309-10337.

11 R. Duan, F. Xia and L. Jiang, ACS Nano, 2013, 7, 8344-8349.

12 F. Lu, S. H. Wu, Y. Hung and C. Y. Mou, Small, 2009, 5, 14081413.

13 M. Bouchoucha, M. F. Côté, R. C. -Gaudreault, M. A. Fortin and F. Kleitz, Chem. Mater., 2016, 28, 4243-4258.

14 R. Jiao, Y. Tan, Y. Jiang, M. Hu, S. Li and Q. Zhai, Ind. Eng. Chem. Res., 2014, 53, 12201-12208.

15 Q. Cai, Z. S. Luo, W. Q. Pang, Y. W. Fan, X. H. Chen and F. Z. Cui, Chem. Mater., 2001, 13, 258-263.

16 Y. D. Chiang, H. Y. Lian, S. Y. Leo, S. G. Wang, Y. Yamauchi and K. C. W. Wu, J. Phys. Chem. C, 2011, 115, 13158-13165.

17 A. Maity, A. Das, D. Sen, S. Mazumder and V. Polshettiwar, Langmuir, 2017, 33, 13774-13782.

18 X. K. Ma, N. H. Lee, H. J. Oh, J. W. Kim, C. K. Rhee, K. S. Park and S. J. Kim, Colloids Surf., A, 2010, 358, 172-176.
19 Y. Li, B. P. Bastakoti, M. Imura, J. Tang, A. Aldalbahi, N. L. Torad and Y. Yamauchi, Chem.-Eur. J., 2015, 21, 6375-6380.

20 E. Santini, J. Krägel, F. Ravera, L. Liggieri and R. Miller, Colloids Surf., A, 2011, 382, 186-191.

21 E. Y. Bryleva, N. A. Vodolazkaya, N. O. Mchedlov-Petrossyan, L. V. Samokhina, N. A. Matveevskaya and A. V. Tolmachev, J. Colloid Interface Sci., 2007, 316, 712-722.

22 W. Wang, B. Gu, L. Liang and W. A. Hamilton, J. Phys. Chem. B, 2004, 108, 17477-17483.

23 T. P. Goloub, L. K. Koopal, B. H. Bijsterbosch and M. P. Sidorova, Langmuir, 2002, 12, 3188-3194.

24 R. Atkin, V. S. J. Craig, E. J. Wanless and S. Biggs, Adv. Colloid Interface Sci., 2003, 316, 712-722.

25 R. Atkin, V. S. J. Craig and S. Biggs, Langmuir, 2000, 16, 93749380.

26 H. Sepehrian, J. Fasihi and M. K. Mahani, Ind. Eng. Chem. Res., 2009, 48, 6772-6775.

27 Y. X. Zhao, M. Y. Ding and D. P. Chen, Anal. Chim. Acta, 2005, 542, 193-198.

28 G. A. Blomfield and L. H. Little, Can. J. Chem., 1973, 51, 1771-1781.

29 F. Quadrifoglio and V. Crescenzi, J. Colloid Interface Sci., 1971, 35, 447-459.

30 K. K. Karukstis, D. A. Savin, C. T. Loftus and N. D. D'Angelo, J. Colloid Interface Sci., 1998, 203, 157-163.

31 A. Dutta and R. K. Dutta, Spectrochim. Acta, Part A, 2014, 126, 270-279. 